\title{
Prudent Expectations Equilibrium in Economies with Uncertain Delivery ${ }^{\star}$
}

\author{
João Correia-da-Silva \\ FCT. Faculdade de Economia. Universidade do Porto. PORTUGAL. \\ Carlos Hervés-Beloso \\ RGEA. Facultad de Económicas. Universidad de Vigo. SPAIN.
}

February 13th, 2006

\begin{abstract}
In an economy with private information, we introduce the notion of objects of choice as lists of bundles out of which the market selects one for delivery. This extension of the model of Arrow-Debreu intends to allow the study of options and default. The model does not require agents to have complete information about the space of states, being suited to a context of Knightian uncertainty. Under the assumption that agents are prudent, equilibrium is characterized by the fact that agents consume bundles with the same utility in states that they do not distinguish.
\end{abstract}

Keywords: General equilibrium, Private information, Incomplete information, Knightian Uncertainty, Ambiguity, Options, Default.

${ }^{\star}$ We are grateful to Jacques Drèze, Jean Gabszewicz, Ani Guerdjikova, Jean-François Mertens, Enrico Minelli, Emma Moreno-García, Mário Páscoa, Juan Pablo Torres-Martinez and Nicholas Yannelis for useful comments and encouragement. João Correia-da-Silva (joaocs@sapo.pt) acknowledges support from Fundação para a Ciência e Tecnologia and FEDER. Carlos Hervés-Beloso (cherves@uvigo.es) acknowledges support by Research Grant BEC2003-09067-C04-01 from Ministerio de Educación y Ciencia and FEDER. 


\section{Introduction}

Uncertainty and private information are crucial in modern economies. Agents know that their decisions can lead to different outcomes, depending on the decisions of others, and on the state of the environment. The complexity associated with these issues is such that it cannot be completely captured by any simple model. A realistic goal is to find simple models that give enlightening, although partial, descriptions.

In general equilibrium theory, several proposals have been made regarding the introduction of private information. An important contribution was made by Radner (1968), who restricted agents to consume the same in states of nature that they did not distinguish. With this condition, the model of Arrow and Debreu (1954) could be reinterpreted in a way that took into account each agent's private information.

Many useful contracts are excluded by this restriction, as the trading of options and contracts with default clauses. The consumption of an agent that sells an option may not be the same in states that the agent does not distinguish, because it depends on whether another agent exercises the option or not. For example, an agent that buys a bundle $A$ and sells an option to get $A$ for $B$, is sure of consuming either $A$ or $B$ (depending on whether the option is exercised or not). This is what we call uncertain delivery: the agent has a list of bundles and is certain of consuming one of them.

From the perspective of the agent, a combination of classical contingent contracts, real options and contracts with default can be translated into a list for uncertain delivery. The list specifies a finite set of possible resulting consumption bundles. The selection of the actual bundle to be consumed is made by the market.

For a general treatment of contracts in a private information setting, the notion of incentive compatibility is needed, as Holmström and Myerson (1983) made clear. The consideration of all incentive compatible trade contracts leads to the highest attainable levels of welfare in the presence of private information. The task of 
studying general equilibrium with incentive compatibility constraints was taken up by Prescott and Townsend (1984a, 1984b), who used linear utility functions to avoid the non-convexities of the incentive compatible set. ${ }^{1}$

A downside of this approach lies in the very demanding informational requirements about the economy and its whole state space. How can an agent know that the contract on the table is incentive compatible? This would be possible in a context of imperfect information, with agents knowing the space of states, or being able to transform the economy with incomplete information into one of imperfect information (Harsanyi, 1967). With complete information, the approach based on incentive compatibility is the adequate. But in some contexts, as in large market interactions, this may be considered a strong requirement.

A contract that delivers the same bundle in states of nature that the agent does not distinguish is necessarily incentive compatible. The agent cannot be tricked and receive a different bundle than the one corresponding to the actual state of nature. In the economy of Radner (1968), agents do not need any knowledge about the space of states of the economy. We can assume that they see the states in the same set of their partition of information as a single state. ${ }^{2}$

In the economy with uncertain delivery, agents buy the same lists for uncertain delivery in states that they do not distinguish. Therefore, the impossibility of observing the state of nature does not raise an issue of incentive compatibility. In states that agents do not distinguish, their rights are the same: to receive one of the bundles in the list. We can still assume that they do not have any additional information about the state space, and that they cannot find out whether a contract is incentive compatible or not. This is an advantage of this model relatively to the models based on the incentive compatible trades.

The economy is not common information, agents only know their own characteris-

\footnotetext{
${ }^{1}$ This is consistent with maximization of expected utility in a consumption space that is the set of lotteries over a maximum of $n$ possible bundles.

${ }^{2}$ In the literature, several terms are used to describe this kind of uncertainty between states which cannot be reduced to a prior probability assessment: Knightian uncertainty, true uncertainty, irreducible uncertainty, ambiguity and ignorance.
} 
tics and the prevailing prices. They do not know the endowments and preferences of the others, and aren't able to figure them out. This irreducible incompleteness of information will lead us to an alternative equilibrium concept. Naturally, the outcome should have a lower welfare level relatively to the model in which the economy is common information.

Contracts for uncertain delivery are strongly related to incentive compatible contracts. In a classical contract, a state is announced and the corresponding net trade is made. The contract is incentive compatible if it is in the self-interest of the agents to announce the states truthfully. With contracts for uncertain delivery, states are not announced. Agents simply decide which net trade to make. In spite of this difference, an incentive compatible contract that delivers $x_{1}$ in state $\omega_{1}$ and $x_{2}$ in state $\omega_{2}$ results in the same consumption as a list that delivers $\left(x_{1} \vee x_{2}\right)$ in both states $\omega_{1}$ and $\omega_{2}$. Since the former contract is incentive compatible, the same incentives imply that the list also delivers $x_{1}$ in state $\omega_{1}$ and $x_{2}$ in state $\omega_{2}$.

There is a recent literature on decision under ambiguity. For our purposes it is enough to refer the work of Gilboa and Schmeidler (1989), who axiomatized maxmin expected utility with multiple priors. The general idea is that agents cannot reduce all their uncertainty into risk, and end up with a set of plausible priors instead of a single one. In this setting, they evaluate expected utility using the worst of the priors. ${ }^{3}$ The incorporation of decision making under ambiguity in general equilibrium theory seems to be a worthy goal, and the present paper is a contribution.

We study economies with incomplete and private information using the concept of uncertain delivery. The solution that we propose is a prudent expectations equilibrium. With objects of choice being lists of bundles out of which the market selects one for delivery, agents expect to receive the worst of the possibilities specified in a list. This leads them to select bundles with the same utility for consumption in states that they do not distinguish, and therefore to obtain the

\footnotetext{
${ }^{3}$ This corresponds to infinite uncertainty aversion in the more general decision making model under ambiguity of Klibanoff, Marinacci and Mukerji (2005).
} 
same utility in states of nature that they do not distinguish. It is a weaker restriction than equal consumption, therefore, the efficiency of trade and welfare are improved relatively to the model of Radner (1968). On the other hand, the welfare of an incentive compatible solution isn't reached. This gives an indication of the welfare cost of ambiguity.

In sum, we model economies with incomplete and private information, in which agents are assumed to be prudent. The model of Arrow-Debreu is reinterpreted to cover this situation, therefore, many classical results still hold: existence of core and equilibrium, core convergence, continuity properties, etc.

The paper is organized as follows: in section 2 we introduce the concept of uncertain delivery and its relation with options and contracts with default; in section 3 we discuss and justify prudent preferences over lists; section 4 includes examples that motivate the paper; the model of general equilibrium with uncertain delivery is formalized in section 5 and characterized in section 6 ; in section 7 , concepts of core in economies with uncertain delivery are introduced and commented; in section 8 we conclude the paper with some remarks. 


\section{Options, Default and Uncertain Delivery}

The theory of general equilibrium under uncertainty has developed upon the formulation of objects of choice as contingent consumption claims (Arrow, 1953). Under this formulation, besides being defined by their physical properties and their location in space and time, commodities are also defined by the state of nature in which they are made available. This incorporation of uncertainty in the commodity space allows an interpretation of the Walrasian model that covers the case of uncertainty.

The Arrow-Debreu (1954) economy with uncertainty extends over two time periods. In the first period, agents know their preferences and endowments, which depend on the state of nature. In this ex ante stage, agents trade state-dependent endowments for state-dependent consumption goods. In the second period, the state of nature becomes public information, trade is realized, and consumption takes place.

In the presence of private information, the state of nature does not become public information. In this case, agents have to be careful when trading contingent goods. Consider a seller that offers the following game:

"I will toss a coin. If the result is heads, you receive a bicycle; if it is tails, you don't receive anything."

If it is common information that the agent does not observe the coin toss, this contingent good has no value because the seller is able to report heads and avoid delivery.

Assuming that agents only trade goods contingent on events that they can observe, Radner (1968) extended the model of Arrow and Debreu to the case of private information. ${ }^{4}$ This restriction implies incentive compatibility. Whatever

\footnotetext{
${ }^{4}$ Private information is introduced in the model simply by restricting the agent's consumption set to values that imply equal consumption in states of nature that the agent does not distinguish. Technically, consumption must be $P_{i}$-measurable, where $P_{i}$ is the $\sigma$-algebra that represents the private information of agent $i$.
} 
the state of nature that occurs, agents are always sure about the bundle that will be delivered to them, so they can never be deceived.

But an agent may accept delivery of different bundles in states of nature that she does not distinguish. Suppose that the seller offers a different game:

"I will toss a coin. If the result is heads, you receive a blue bicycle; if it is tails, you receive a red bicycle."

Even if it is common information that the agent does not observe the coin toss, this is a valuable uncertain contingent good, because the delivery of a "bicycle" is guaranteed.

In our model, agents are allowed to buy "contracts for uncertain delivery" or "lists", which specify a list of bundles out of which the market selects one for delivery. These contracts can also be contingent. A "contingent list" gives the right to one of the bundles in the list if the specified contingency occurs.

These objects of choice are more general than contingent consumption claims. ${ }^{5}$ Actually, the possible trades are, in a certain sense, the incentive compatible trades. To see this, consider an incentive compatible contract delivering $x_{1}$ in state $\omega_{1}$ and $x_{2}$ in state $\omega_{2}$. This contract can be substituted by a contract for uncertain delivery of $\left(x_{1} \vee x_{2}\right)$. The fact that the former contract is incentive compatible implies that the contract for uncertain delivery also delivers $x_{1}$ in $\omega_{1}$ and $x_{2}$ in $\omega_{2}$.

Included in the definition of contract for uncertain delivery is the trading of options of a certain kind. Suppose that an agent buys a bundle $x$ while selling an option to make a net trade $y$. The final consumption bundle will be $x$ if the "market" does not exercise the option, and $x-y$ if the option is exercised. The corresponding list for uncertain delivery can be written as $(x \vee x-y)$.

The trading of contracts with default clauses is also covered by this definition. Suppose that an agent buys the bundle $x$, but gives the market the possibility

\footnotetext{
${ }^{5}$ Notice that contracts for contingent delivery (Arrow, 1953) can be seen as "contracts for uncertain delivery" with lists of only one element.
} 
of defaulting the contract and delivering bundle $y$ as a penalty. The resulting uncertain bundle is $(x \vee y)$.

An agent that trades contingent goods, real options and contracts with default, can calculate the list of possible outcomes. The actual outcome will depend on the choice of the market. We put a finite bound on the number of possible outcomes to $\mathrm{a}$ in order to work in a finite dimensional space. The objects of choice in this setting are the lists for uncertain delivery. Allowing contracts for uncertain delivery may be seen as the opening of additional markets, including markets for options and contracts with default. 


\section{Prudent preferences}

With agents selecting among uncertain bundles, it is necessary to extend the domain in which preferences are defined. What is the utility of receiving " $x_{1}$ or $x_{2}$ "? In this paper we use maxmin preferences (Gilboa and Schmeidler, 1989). ${ }^{6}$ Agents attribute to the uncertain bundle $\left(x_{1} \vee x_{2}\right)$ the utility of the worst possibility:

$$
U\left(x_{1} \vee x_{2}\right)=\min \left\{u\left(x_{1}\right), u\left(x_{2}\right)\right\} .
$$

Contingent bundles which are constant in states that the agent does not distinguish can be seen as contingent lists with only one element. In this case, prudent utility is equal to the usual expected utility. This way, the utility functions are extended to a domain that also includes lists, but the values in the original consumption space are preserved.

The motivation for the use of prudent preferences is essentially the same of the literature on decision making under ambiguity (Schmeidler, 1989). Agents are assumed to be completely ignorant with respect to the differences between states that their information structures do not allow them to distinguish. Therefore, they cannot reduce their uncertainty about which of the bundles in the list will be delivered into a simple prior.

Consider, for example, a seller which has a "bicycle" to sell. Instead of selling a "bicycle", the seller could gain by selling a "bicycle or car", and always deliver the "bicycle" and never the car. In order to avoid being deceived by informed sellers, the agents behave defensively, expecting the worst outcome.

If the bundles are actually portfolios that give a money return, then it is realistic to consider that the worst possibility is going to be delivered. Suppose that there is an additional round of trade. Agents should evaluate the utility of bundles (portfolios) by their value in the second round of trade. Sellers do exactly the same valuation. In this case, the seller always delivers the bundle with the lowest (ex-post) value, so this worst bundle is what the buyer always receives.

\footnotetext{
${ }^{6}$ These preferences can also be seen as a degenerate case of Choquet expected utilities (Schmeidler, 1989) since the whole weight is placed on the worst possible utility.
} 
In this section we provide two complementary justifications for prudent behavior. First, prudence is shown to result from two apparently reasonable assumptions. In a second subsection, we suggests that prudence is not such a strong assumption when the market is assumed to know the information of the agents, and to customize lists in the way that maximizes their utility for the agent. This seems to justify the adoption of defensive behavior.

\subsection{Prudence as a result}

The following analysis resembles the work of Arrow and Hurwicz (1972) on optimality criteria for decision making under ignorance. By "ignorance" it is meant that the agent has no prior probabilities on the occurrence of different states, perceiving them as a single state.

Only the "prudent preferences" satisfy the two assumptions made precise below. The first is a kind of independence, and the second is related to realism. According to the first, substituting one possibility in a list by another that has greater utility does not make the list less attractive.

(P1) $x_{j} \succeq y_{j}, \forall j=1, \ldots, k \Rightarrow\left(x_{1} \vee \ldots \vee x_{k}\right) \succeq\left(y_{1} \vee \ldots \vee y_{k}\right)$

Suppose that an agent is indifferent between receiving a "blue bicycle" and a "red bicycle". What utility should be assigned to the delivery of a "blue bicycle or red bicycle"? Some agents may prefer to know what will be delivered in the future, while others may prefer to be surprised. A corollary of $P 1$ is that agents are neutral with respect to surprise:

$$
\forall x_{1}, \ldots, x_{k}: x_{1} \sim \ldots \sim x_{k} \Rightarrow\left(x_{1} \vee \ldots \vee x_{k}\right) \sim x_{1} \sim \ldots \sim x_{k}
$$

It is easy to see that $P 1$ implies that the utility of a list cannot be lower than the utility of the worst possibility. Assuming that the least preferred bundle is $x_{1}$ :

$$
x_{j} \succeq x_{1}, \forall j=1, \ldots, k \Rightarrow\left(x_{1} \vee \ldots \vee x_{k}\right) \succeq\left(x_{1} \vee \ldots \vee x_{1}\right) \sim x_{1}
$$

The second property means that an agent is indifferent between a bundle $x_{1}$ and a list with $x_{1}$ and $x_{1}+a$, where $a \geq 0$. If the seller keeps the contract by 
delivering $x_{1}$, why would she make the effort to deliver the additional $a$ ? The agent realistically expects to receive always $x_{1}$, and never $x_{1}+a$.

(P2) $\forall x_{1}, \ldots, x_{k}: x_{k+1} \geq x_{k} \Rightarrow\left(x_{1} \vee \ldots \vee x_{k} \vee x_{k+1}\right) \sim\left(x_{1} \vee \ldots \vee x_{k}\right)$.

To see that $P 1$ and $P 2$ together imply maxmin preferences, assume that preferences satisfy $P 1$ and $P 2$. Introducing an alternative such as $x_{k+1} \geq x_{k}$ does not increase the utility of the list, and, by $P 1$, introducing an alternative that is less attractive than $x_{k+1}$ also does not. Thus there isn't any additional alternative that increases the utility of the list.

$$
\forall x_{1}, \ldots, x_{k}, x_{k+1}:\left(x_{1} \vee \ldots \vee x_{k} \vee x_{k+1}\right) \preceq\left(x_{1} \vee \ldots \vee x_{k}\right)
$$

Seeing lists as a combination of contingent contracts and options, the last property implies that offering an option never increases utility.

Under $P 1$ and $P 2$, agents behave with prudence, being indifferent between the uncertain bundle and the worst possibility:

$$
\begin{aligned}
& x_{1} \preceq x_{j}, \forall j=1, \ldots, k \Rightarrow\left(x_{1} \vee \ldots \vee x_{k}\right) \sim x_{1} \Leftrightarrow \\
& \Leftrightarrow \forall x_{1}, \ldots, x_{k}: u\left(x_{1} \vee \ldots \vee x_{k}\right)=\min _{j=1, \ldots, k} u\left(x_{j}\right) .
\end{aligned}
$$

\subsection{Prudence by construction}

Suppose that the market knows the preferences and information of the agent. Instead of selling a bundle, the market will prefer to offer the list that includes this bundle but has more utility. This suggests a transformation of the preferences. For each bundle, consider the utility of the best list that contains this bundle (or list).

The justification for this is based on the hypothesis that the market is informed about the preferences of the buyer, and sells a bundle as the most attractive list that contains it. Suppose that the market has a bicycle to offer. If the buyer prefers a "car or bicycle" to a bicycle, then the market will always offer a "car or 
bicycle". If this is the most attractive way to sell the bicycle, then the indirect utility of the bicycle is equal to the utility of the "car or bicycle".

Consider the utility function of agent $i, v_{i}$, describing preferences over lists. Find the indirect utility of a list, $v_{i}^{\prime}\left(\tilde{x}_{1}\right)$, as the maximum utility of a list containing $l x_{1}:{ }^{7}$

$$
v_{i}^{\prime}\left(\tilde{x}_{1}\right)=\max \left\{v_{i}\left(\tilde{x}_{1} \vee \ldots\right)\right\} .
$$

Knowing the preferences of the agent, the market sells $\tilde{x}_{1}$ as the most attractive list containing $\tilde{x}_{1}$. If the inclusion of additional alternatives increases utility for the buyer, the seller will include them. In any case, $\tilde{x}_{1}$ will be delivered, but the buyer does not know this. Observe that under the assumption of prudent preferences, we have $v_{i}^{\prime}=v_{i}$.

If the seller has a product to deliver which is $\left(x_{1} \vee x_{2}\right)$, then this product cannot have more indirect utility than either $x_{1}$ or $x_{2}$. Notice that the seller has to sell this product as a list containing $\left(x_{1} \vee x_{2}\right)$, which cannot have more indirect utility than all the lists containing $x_{1}$ or $x_{2}$. The inclusion of additional alternatives does not increase the indirect utility of the list:

$$
\text { (A) } v^{\prime}\left(x_{1} \vee x_{2}\right)=\max \left\{v\left(x_{1} \vee x_{2} \vee \ldots\right)\right\} \leq \min \left\{v^{\prime}\left(x_{1}\right), v^{\prime}\left(x_{2}\right)\right\}
$$

It is reasonable to assume that, faced with the possibility of receiving the list $\tilde{x}_{1}$ or the list $\tilde{x}_{2}$, the agents do not prefer to receive the worst list with certainty.

$$
v\left(l x_{1} \vee l x_{2}\right) \geq \min \left\{v\left(l x_{1}\right), v\left(l x_{2}\right)\right\} .
$$

Denote by $\tilde{y}$ a list containing both $\tilde{x}_{1}$ and $\tilde{x}_{2}$ that maximizes indirect utility, that is, such that $v(\tilde{y})=v^{\prime}\left(\tilde{x}_{1} \vee \tilde{x}_{2}\right)$. Similarly, find the list $\tilde{y}_{1}$ such that $v\left(\tilde{y}_{1}\right)=v^{\prime}\left(\tilde{x}_{1}\right)$ and the list $\tilde{y}_{2}$ such that $v\left(\tilde{y}_{2}\right)=v^{\prime}\left(\tilde{x}_{2}\right)$. Since $\tilde{y}$ is a maximizer:

$$
v(\tilde{y}) \geq v\left(\tilde{y}_{1} \vee \tilde{y}_{2}\right) \geq \min \left\{v\left(\tilde{y}_{1}\right), v\left(\tilde{y}_{2}\right)\right\} \Leftrightarrow v^{\prime}\left(\tilde{x}_{1} \vee \tilde{x}_{2}\right) \geq \min \left\{v^{\prime}\left(\tilde{x}_{1}\right), v^{\prime}\left(\tilde{x}_{2}\right)\right\} .
$$

Together with (A), this implies that the transformed preferences are prudent:

$$
v^{\prime}\left(x_{1} \vee x_{2}\right)=\min \left\{v^{\prime}\left(x_{1}\right), v^{\prime}\left(x_{2}\right)\right\} .
$$

\footnotetext{
${ }^{7}$ Here $\tilde{x}_{1}$ denotes a list, and $\tilde{x}_{1} \vee \ldots$ denotes any list containing $\tilde{x}_{1}$.
} 
Thus, with respect to indirect utility, prudence seems to be a weak restriction. But for indirect utility to be useful in our model, it is necessary that it is also continuous, weakly monotonic, and concave. It is reasonable to accept that continuity and weak monotonicity are preserved. The assumption of concaveness is harder to interpret.

$$
\begin{aligned}
& v^{\prime}\left(\lambda x_{1}+(1-\lambda) x_{2}\right) \geq \lambda v^{\prime}\left(x_{1}\right)+(1-\lambda) v^{\prime}\left(x_{2}\right) \Leftrightarrow \\
& \Leftrightarrow \max \left\{v\left(\lambda x_{1}+(1-\lambda) x_{2} \vee \ldots\right)\right\} \geq \lambda \max \left\{v\left(x_{1} \vee \ldots\right)\right\}+(1-\lambda) \max \left\{v\left(x_{2} \vee \ldots\right)\right\} .
\end{aligned}
$$

Having the lottery, $\lambda x_{1}+(1-\lambda) x_{2}$, the seller can: (1) sell it as one of the elements of a list with utility $v^{\prime}\left(\lambda x_{1}+(1-\lambda) x_{2}\right)$; or (2) sell it as a lottery between two lists. One that gives $v^{\prime}\left(x_{1}\right)$, and other that gives $v^{\prime}\left(x_{2}\right)$. Concaveness of $v^{\prime}$ means that agents weakly prefer the first "package".

Under these hypothesis, prudence is guaranteed by construction. With the market knowing the preferences of the agent, it is easier to accept the assumption of prudent preferences made in this paper. 


\section{Examples}

In this section, we give two examples of trading between two agents who do not have any common information. As a result, if agents are constrained to consume the same in states that they do not distinguish, there is no trade in equilibrium. With contracts for uncertain delivery, agents can trade and welfare improves in the sense of Pareto.

\section{Example 1: Perfect substitutes}

This economy has two agents and four commodities: "ham sandwiches", "cheese sandwiches", "orange juices" and "apple juices". Sandwiches are perfect substitutes, as well as the juices. Agents want to eat and drink. Precisely, they maximize expected utility, having state-contingent preferences described by a Cobb-Douglas utility function:

$$
u=\left(s_{h}+s_{c}\right)^{0.5} \cdot\left(j_{o}+j_{a}\right)^{0.5} .
$$

There are four possible states of nature, $\Omega=\left\{\omega_{1}, \omega_{2}, \omega_{3}, \omega_{4}\right\}$.

- In $\omega_{1}$, agent $A$ is endowed with two "ham sandwiches" and agent $B$ with two "orange juices": $e_{A}\left(\omega_{1}\right)=(2,0,0,0)$ and $e_{B}\left(\omega_{1}\right)=(0,0,2,0)$;

- In $\omega_{2}$, agent $A$ is endowed with two "ham sandwiches" and agent $B$ with two "apple juices": $e_{A}\left(\omega_{2}\right)=(2,0,0,0)$ and $e_{B}\left(\omega_{2}\right)=(0,0,0,2)$;

- In $\omega_{3}$, agent $A$ is endowed with two "cheese sandwiches" and agent $B$ with two "orange juices": $e_{A}\left(\omega_{3}\right)=(0,2,0,0)$ and $e_{B}\left(\omega_{3}\right)=(0,0,2,0)$;

- In $\omega_{4}$, agent $A$ is endowed with two "cheese sandwiches" and agent $B$ with two "apple juices": $e_{A}\left(\omega_{4}\right)=(0,2,0,0)$ and $e_{B}\left(\omega_{4}\right)=(0,0,0,2)$.

Each agent observes only its endowments. The information partitions are:

$$
P_{A}=\left\{\left\{\omega_{1}, \omega_{2}\right\},\left\{\omega_{3}, \omega_{4}\right\}\right\} \text { and } P_{B}=\left\{\left\{\omega_{1}, \omega_{3}\right\},\left\{\omega_{2}, \omega_{4}\right\}\right\} .
$$


Agents want to guarantee that they will eat and drink in the future. The problem is that they are unable to make trades contingent upon events (sets of states) that they observe. For example, agent $A$ would like to consume orange juice. For consumption to be the same across undistinguished states, the delivery of orange juice must be the same in $\omega_{1}$ and $\omega_{2}$ and the same in $\omega_{3}$ and $\omega_{4}$. This is not possible, because agent $B$ only has orange juice in the states $\omega_{1}$ and $\omega_{3}$. The same reasoning applies to each of the other commodities, so there is no trade in the economy. ${ }^{8}$

Nevertheless, contracts for uncertain delivery allow agents to guarantee future consumption of a sandwich and a juice. The agents can agree to deliver a "sandwich" for a "juice" in the second period. This constitutes a contract for uncertain delivery in which one of the agents gets the right to receive an "orange juice or apple juice", while the other gets the right to receive a "ham sandwich or cheese sandwich".

Delivery of an "orange juice or apple juice" may be seen as a contract with a default clause, in which the agent should deliver an "orange juice", but may default and pay an "apple juice" as penalty. It may also be seen as the combination of a contract for the delivery of an "orange juice", with an option to get the "orange juice" in exchange for an "apple juice".

Since agent $A$ is able to ensure the delivery of a sandwich and agent $B$ is able to ensure the delivery of a juice, this contract for uncertain delivery allows them to attain the optimal outcome:

$$
x_{A}=x_{B}=\left\{\begin{array}{l}
(1,0,1,0) \text { in } \omega_{1}, \\
(1,0,0,1) \text { in } \omega_{2}, \\
(0,1,1,0) \text { in } \omega_{3}, \\
(0,1,0,1) \text { in } \omega_{4} .
\end{array}\right.
$$

Both agents obtain an utility that is equal to 1 in all states of nature. This constitutes an improvement in the sense of Pareto relatively to the Walrasian

\footnotetext{
${ }^{8}$ It should be clear that we can assume strictly positive endowments, substituting every zero for a small $\epsilon$, and reach similar conclusions.
} 
expectations equilibrium solution, which resulted in an utility of zero to both agents.

\section{Example 2 - Risk sharing}

Consider an economy with two agents, two goods and three states of nature. Initial endowments depend on the state of nature:

$$
e_{A}=\left\{\begin{array}{l}
(199,100) \text { in } \omega_{1}, \\
(1,100) \text { in }\left\{\omega_{2}, \omega_{3}\right\} .
\end{array} \quad e_{B}=\left\{\begin{array}{l}
(1,100) \text { in }\left\{\omega_{1}, \omega_{2}\right\} \\
(199,100) \text { in } \omega_{3} .
\end{array}\right.\right.
$$

The state $\omega_{2}$ has a probability of $0,2 \%$, while $\omega_{1}$ and $\omega_{3}$ have a probability of 49,9\%. Agents know these probabilities but there are no commonly observed events:

$$
P_{A}=\left\{\left\{\omega_{1}\right\},\left\{\omega_{2}, \omega_{3}\right\}\right\} \text { and } P_{B}=\left\{\left\{\omega_{1}, \omega_{2}\right\},\left\{\omega_{3}\right\}\right\} .
$$

Agents want to maximize expected utility, having the same preferences in all states of nature. The marginal utility of good 1 is decreasing, while marginal utility of good 2 is constant:

$$
u_{A}\left(x_{1}, x_{2}\right)=u_{B}\left(x_{1}, x_{2}\right)=10 \sqrt{x_{1}}+x_{2} .
$$

Observe the symmetry in the economy: agent $A$ wants to sell good 1 in $\omega_{1}$ and to buy in $\left\{\omega_{2}, \omega_{3}\right\}$, while agent $B$ wants to buy in $\left\{\omega_{1}, \omega_{2}\right\}$ and to sell in $\omega_{3}$.

The total resources in the economy are:

$$
e_{\text {total }}=\left\{\begin{array}{l}
(200,200) \text { in } \omega_{1}, \\
(2,200) \text { in } \omega_{2}, \\
(200,200) \text { in } \omega_{3}
\end{array}\right.
$$

Restricting consumption to be the same in states that agents do not distinguish, Pareto improvements relatively to the initial endowments are not possible. But, as in example 1, contracts for uncertain delivery allow agents to improve their welfare.

With prudent preferences, the equilibrium allocation is: 


$$
\begin{aligned}
& x^{A}=\left\{\begin{array}{l}
(100,190) \text { in } \omega_{1}, \\
(1,100) \text { in } \omega_{2}, \\
(100,10), \omega_{3} .
\end{array}\right. \\
& x^{B}=\left\{\begin{array}{l}
(100,10) \text { in } \omega_{1}, \\
(1,100) \text { in } \omega_{2}, \\
(100,190), \omega_{3} .
\end{array} \quad u^{B}=\left\{\begin{array}{l}
290 \text { in } \omega_{1}, \\
110 \text { in }\left\{\omega_{2}, \omega_{3}\right\} .
\end{array}\right.\right.
\end{aligned}
$$

Agents can obtain this allocation by trading an option. In every state of nature, each agent has the option to get 90 units of good 2 in exchange for 99 units of good 1. This is equivalent to uncertain delivery of $(99,-90)$ or $(0,0)$. It is straightforward to see that agent A exercises the option in state 1 and agent B exercises the option in state 3 . 


\section{General Equilibrium with Uncertain Delivery}

The model of the economy with uncertain delivery is based on the model known as a differential information economy, but with a modified informational restriction. Agents must select the same list for delivery in states that they do not distinguish, instead of the same bundle. Technically, the list must be $P_{i}$-measurable, which does not imply that consumption is $P_{i}$-measurable. ${ }^{9}$.

Consider three possible states of nature, $\Omega=\left\{\omega_{1}, \omega_{2}, \omega_{3}\right\}$, and an agent who does not distinguish $\omega_{1}$ from $\omega_{2}$. Instead of observing prices in $\omega_{1}, \omega_{2}$ and $\omega_{3}$, the agent only observes prices for delivery in the events $\left\{\omega_{1}, \omega_{2}\right\}$ and $\omega_{3}$. In the economy with uncertain delivery, the market also reports the prices of lists. Denote the price of the list $\left(x_{1} \vee x_{2}\right)$ in $\omega_{1}$ by $\tilde{p}_{1}\left(x_{1} \vee x_{2}\right)$. The agent only observes the price of $\left(x_{1} \vee x_{2}\right)$ in the event $\left\{\omega_{1}, \omega_{2}\right\}$, that is, $\tilde{p}_{1}\left(x_{1} \vee x_{2}\right)+\tilde{p}_{2}\left(x_{1} \vee x_{2}\right)$.

Suppose that an agent buys the list $\tilde{x}=\left[\left(x_{1} \vee x_{2}\right),\left(x_{1} \vee x_{2}\right), x_{3}\right]$. To keep this contract for uncertain delivery, the market must deliver one of the four contingent bundles: $\left(x_{1}, x_{1}, x_{3}\right),\left(x_{1}, x_{2}, x_{3}\right),\left(x_{2}, x_{1}, x_{3}\right),\left(x_{2}, x_{2}, x_{3}\right)$. It is as if a fictitious intermediary had to guarantee delivery of one of the alternatives. The intermediary would buy the cheapest contingent bundle satisfying the requirements of the list and deliver it to the agent. For the list to be deliverable, its price cannot be lower than the price of the cheapest alternative.

$$
\tilde{p}_{1}\left(x_{1} \vee x_{2}\right)+\tilde{p}_{2}\left(x_{1} \vee x_{2}\right) \geq \min \left\{p_{1} \cdot x_{1}, p_{1} \cdot x_{2}\right\}+\min \left\{p_{2} \cdot x_{1}, p_{2} \cdot x_{2}\right\} .
$$

The price of the list $\left(x_{1} \vee x_{2}\right)$ in the event $\left\{\omega_{1}, \omega_{2}\right\}$ cannot be higher than the price of the cheapest alternative. If it were, there would be an opportunity for arbitrage. Another intermediary could buy the cheapest alternative and sell the list with profit. This no-profit condition prevents prices of options from being negative. $^{10}$

$$
\tilde{p}_{1}\left(x_{1} \vee x_{2}\right)+\tilde{p}_{2}\left(x_{1} \vee x_{2}\right) \leq \min \left\{p_{1} \cdot x_{1}, p_{1} \cdot x_{2}\right\}+\min \left\{p_{2} \cdot x_{1}, p_{2} \cdot x_{2}\right\}
$$

\footnotetext{
${ }^{9}$ In their study of implementation in differential information economies, Postlewaite and Schmeidler (1986) do not impose any measurability restriction on the outcome

${ }^{10}$ Observe that a list $(A \vee B)$ can be seen obtained by buying bundle $A$ and selling an option to get $A$ for $B$.
} 
In sum, given the prices of the contingent goods, the supply curve of a list is a vertical line passing on the price of the cheapest contingent bundle satisfying the requirements of the list. The price of a list is equal to the minimum of the prices of the contingent bundles satisfying its requirements of the list. This gives a unique extension of prices from the space of goods to the space of lists. The price of the list $\left(x_{1} \vee \ldots \vee x_{k}\right)$ to be delivered in state $\omega_{s}$ is:

$$
\tilde{p}_{s}\left(x_{1} \vee \ldots \vee x_{k}\right)=\min _{j=1, \ldots, k} p_{s} \cdot x_{j}
$$

With this restriction on the prices of lists, it is enough to find equilibrium prices for the contingent commodities. The prices of the lists follow directly from those.

When an agent buys a list, the cheapest bundle satisfying the requirements of the lists plays a fundamental role. It is the bundle that chosen for delivery, and, furthermore, its price is equal to the price of the list.

Finding the bundles actually delivered by the market allows us to find equilibrium lists selected by the agents. From all the lists compatible with the given delivery, the list selected by a maximizing agent is the most preferred one, since all of them have the same price (the price of the delivered bundle).

With prudent preferences, we know that additional alternatives never increase the utility of a list. Then, the list selected for a given state can be constructed using the alternatives delivered in states that the agent does not distinguish from this state. For example, an agent may receive a consumption bundle that is not $P_{i}$-measurable, $x=\left(x_{1}, x_{2}, x_{3}\right)$. It can be induced that this would be the result of buying the $P_{i}$-measurable list $\tilde{x}=\left[\left(x_{1} \vee x_{2}\right),\left(x_{1} \vee x_{2}\right), x_{3}\right]$. No other list compatible with the delivery of $x_{i}$ has strictly higher utility.

The economy extends over two time periods. In the first, agents trade their statecontingent endowments for state-contingent lists. In the second period agents receive (and consume) one of the bundles that corresponds to a state that they do not distinguish from the actual state of nature.

Consider a finite number of agents, commodities and states of nature. In the economy with uncertain delivery, $\mathcal{E} \equiv\left(e_{i}, u_{i}, P_{i}, q_{i}\right)_{i=1}^{n}$, for each agent $i$ : 
- A partition of $\Omega, P_{i}$, represents the private information of the agent. Sets that belong to $P_{i}$ are denoted $A_{i}^{j}$. The set of states of nature that agent $i$ does not distinguish from $\omega_{k}$ is denoted $P_{i}\left(\omega_{k}\right)$.

- Agents assign subjective probabilities to the different elementary events that they observe. To each set $A_{i}^{j} \in P_{i}$ corresponds a prior probability $\operatorname{Pr}_{i}\left(A_{i}^{j}\right)$, with $\sum_{j} \operatorname{Pr}_{i}\left(A_{i}^{j}\right)=1$.

- Preferences over consumption bundles are the same in undistinguished states, represented by the Von Neumann-Morgenstern (1944) utility functions $u_{i}^{j}: \mathbb{R}_{+}^{l} \rightarrow \mathbb{R}_{+}$, which are assumed to be continuous, weakly monotone and concave.

- The initial endowments are constant across undistinguished states, and strictly positive: $e_{i}^{j} \gg 0$ for all $j$.

The objective function, prudent expected utility, is equal to the expected interim utility:

$$
\tilde{U}_{i}\left(\tilde{x}_{i}\right)=\sum_{A_{i}^{j} \in P_{i}} \operatorname{Pr}_{i}\left(A_{i}^{j}\right) \tilde{v}_{i}^{A_{i}^{j}}\left(\tilde{x}_{i}^{A_{i}^{j}}\right)=\sum_{\omega_{j} \in \Omega} q_{i}^{j} \tilde{v}_{i}^{j}\left(\tilde{x}_{i}^{j}\right) .
$$

Knowing the bundle that the agent receives, we know that the utility of the list that the agent bought is the same. It could not be higher, because the agent evaluates the list according to the worst alternative. If it were lower, then the agent receiving $x_{i}$ would not be maximizing. The agent would be better off selecting the list:

$$
\tilde{x}_{i}^{s}=\bigvee_{\omega_{s} \in P_{i}\left(\omega_{s}\right)} x_{i}^{s}
$$

The utility of this list is the same as the bundle delivered, and has the same price (remember the equality between the prices of lists and the prices of the actually delivered bundles). Since the price and the utility of a list is equal to the price and utility of the delivered bundle, the problem of the consumer can be written on bundles instead of lists. The objective function is the expected interim utility:

$$
U_{i}\left(x_{i}\right)=\sum_{A_{i}^{j} \in P_{i}} \operatorname{Pr}_{i}\left(A_{i}^{j}\right) v_{i}^{j}\left(x_{i}\right) .
$$


Prudence is incorporated in the interim utility. Agent $i$ knows that the state of nature that occurred belongs to some $A_{i}^{j}$, and that she has the right to receive one of the bundles $x_{i}^{s}$ with $\omega_{s} \in A_{i}^{j}$. Under prudent expectations, the utility that the agent expects corresponds to the worst possibility:

$$
v_{i}^{j}\left(x_{i}\right)=\min _{\omega_{s} \in A_{i}^{j}} u_{i}^{j}\left(x_{i}^{s}\right){ }^{11}
$$

From the properties of the state-dependent utility functions, $u_{i}^{j}$, it is shown below that the prudent expected utility function is also concave.

$$
\begin{aligned}
& U_{i}\left(\lambda x_{i}+(1-\lambda) y_{i}\right)=\sum_{A_{i}^{j} \in P_{i}} q_{i}^{j} v_{i}^{j}\left(\lambda x_{i}+(1-\lambda) y_{i}\right)= \\
= & \sum_{A_{i}^{j} \in P_{i}} q_{i}^{j} \min _{\omega \in A_{i}^{j}}\left\{u_{i}^{j}\left(\lambda x_{i}(\omega)+(1-\lambda) y_{i}(\omega)\right)\right\} \geq \\
\geq & \sum_{A_{i}^{j} \in P_{i}} q_{i}^{j} \min _{\omega \in A_{i}^{j}}\left\{\lambda u_{i}^{j}\left(x_{i}(\omega)\right)+(1-\lambda) u_{i}^{j}\left(y_{i}(\omega)\right)\right\} \geq \\
\geq & \sum_{A_{i}^{j} \in P_{i}} q_{i}^{j} \min _{\omega \in A_{i}^{j}}\left\{\lambda u_{i}^{j}\left(x_{i}(\omega)\right)\right\}+\sum_{A_{i}^{j} \in P_{i}} q_{i}^{j} \min _{\omega \in A_{i}^{j}}\left\{(1-\lambda) u_{i}^{j}\left(y_{i}(\omega)\right)\right\}= \\
= & \lambda \sum_{A_{i}^{j} \in P_{i}} q_{i}^{j} v_{i}^{j}\left(x_{i}\right)+(1-\lambda) \sum_{A_{i}^{j} \in P_{i}} q_{i}^{j} v_{i}^{j}\left(y_{i}\right)= \\
= & \lambda U_{i}\left(x_{i}\right)+(1-\lambda) U_{i}\left(y_{i}\right) .
\end{aligned}
$$

Therefore, the model of Arrow-Debreu can be reinterpreted to cover the case of an economy with uncertain delivery in which agents are prudent. The economy with uncertain delivery is equivalent to an Arrow-Debreu economy, $\mathcal{E}_{A D} \equiv\left(e_{i}, U_{i}\right)_{i=1}^{n}$, where, for each agent $i$ :

- Preferences are described by an utility function, $U_{i}: \mathbb{R}_{+}^{\Omega l} \rightarrow \mathbb{R}_{+}$, that is continuous, weakly monotone and concave. It is defined as $U_{i}\left(x_{i}\right)=\sum_{A_{i}^{j} \in P_{i}} \operatorname{Pr}_{i}\left(A_{i}^{j}\right) v_{i}^{j}\left(x_{i}\right)=\sum_{A_{i}^{j} \in P_{i}} \operatorname{Pr}_{i}\left(A_{i}^{j}\right) \min _{\omega \in A_{i}^{j}} u_{i}^{j}\left(x_{i}(\omega)\right)$.

- The vector of initial endowments, $e_{i} \in \mathbb{R}_{+}^{\Omega l}$, is strictly positive.

\footnotetext{
${ }^{11}$ Note that if $x$ is equal across states that the agent does not distinguish, then prudent expected utility is equal to classical expected utility. If agents are perfectly informed this always occurs. With symmetric information, the transformed model is equivalent to the classical model of Arrow and Debreu.
} 
With the economy formalized as an Arrow-Debreu economy, the usual definitions apply. An allocation, $x$, is feasible if and only if:

$$
\sum x \leq \sum e \Leftrightarrow \forall \omega: \sum x(\omega) \leq \sum e(\omega) .
$$

We normalize the price functions to the simplex of $\mathbb{R}^{\Omega l}$, that is:

$$
\Delta=\left\{p \in \mathbb{R}^{\Omega l}: \sum_{\omega \in \Omega} \sum_{k=1, \ldots, l} p_{k}(\omega)=1\right\} .
$$

The "budget set" of agent $i$ is given by:

$$
B_{i}\left(p, e_{i}\right)=\left\{x_{i} \in \mathbb{R}^{\Omega l} \text {, such that } \sum_{\Omega} p(\omega) x_{i}(\omega) \leq \sum_{\Omega} p(\omega) e_{i}(\omega)\right\} .
$$

A pair $\left(p^{*}, x^{*}\right)$ is a Walrasian equilibrium with prudent expectations if $p^{*}$ is a price system and $x^{*}=\left(x_{1}^{*}, \ldots, x_{n}^{*}\right)$ is a feasible allocation such that, for every $i, x_{i}^{*} \in \mathbb{R}_{+}^{\Omega l}$ maximizes prudent expected utility, $U_{i}$, on $B_{i}\left(p^{*}, e_{i}\right)$.

Private information was introduced in the model of Arrow and Debreu by a transformation of the preferences. This transformation preserves the properties of continuity, weak monotonicity and concaveness. Therefore, several classical results still hold: existence of core and Walrasian equilibrium, core convergence, continuity properties, etc. ${ }^{12}$

The following theorem can be stated without proof.

Theorem 1 There exists a Walrasian equilibrium with prudent expectations.

As seen before, from the equilibrium defined on allocations, $\left(x^{*}, p^{*}\right)$, we can determine the equilibrium defined on the space of lists, $\left(\tilde{x}^{*}, \tilde{p}^{*}\right)$.

\footnotetext{
${ }^{12}$ This framework also allows the analysis of continuity properties of equilibrium with respect to information as a problem of continuity with respect to preferences, which was settled by Hildenbrand and Mertens (1972).
} 


\section{Characteristics of Equilibrium}

\section{Utility is $P_{i}$-measurable}

In this extended Arrow-Debreu model, equilibrium allocations are characterized by the fact that in states of nature that an agent does not distinguish, the utility of the delivered bundles tends to be equal. Instead of imposing consumption to be $P_{i}$-measurable, as Radner (1968) and Yannelis (1991), we see a weaker restriction of $P_{i}$-measurable utility arising naturally.

If equilibrium prices of the contingent commodities aren't strictly positive, then the utility of some equilibrium allocations may differ across undistinguished states. But we can refine the equilibrium set by removing a free component of excess supply. With the same equilibrium prices, the resulting equilibrium allocations have $P_{i}$-measurable utility. The following results make this precise.

Theorem 2 Let $\left(x^{*}, p^{*}\right)$ be a Walrasian equilibrium with prudent expectations. Then, for each agent $i, x_{i}^{*}=y_{i}^{*}+z_{i}$, with $y_{i}^{*}$ having the same utility in states that the agent does not distinguish, and $z_{i}$ being "free". Precisely:

$$
\begin{aligned}
& y_{i}^{*} \in \mathbb{R}_{+}^{\Omega l} \text { and such that: } \omega^{\prime} \in P_{i}(\omega) \Rightarrow u_{i}^{\omega}\left(y_{i}^{*}(\omega)\right)=u_{i}^{\omega^{\prime}}\left(y_{i}^{*}\left(\omega^{\prime}\right)\right) . \\
& z_{i} \in \mathbb{R}_{+}^{\Omega l} \text { and such that: } p^{*} \cdot z_{i}=0 .
\end{aligned}
$$

Proof. Recall that for any $\omega^{\prime} \in P_{i}(\omega)$, preferences are the same: $u_{i}^{\omega}=u_{i}^{\omega^{\prime}}$. Now suppose that for some $\omega^{\prime} \in P_{i}(\omega)$, we have different utilities, that is: $u_{i}^{\omega}\left(x_{i}^{*}(\omega)\right)>$ $u_{i}^{\omega}\left(x_{i}^{*}\left(\omega^{\prime}\right)\right)$. Then, there exists some $\delta<1$ such that $u_{i}^{\omega}\left(\delta \cdot x_{i}^{*}(\omega)\right)=u_{i}^{\omega}\left(x_{i}^{*}\left(\omega^{\prime}\right)\right)$. Whenever this occurs, modify the allocation accordingly to obtain $y_{i}^{*} \leq x_{i}^{*}$. This allocation has $P_{i}$-measurable utility. If $y_{i}^{*}$ belongs to the interior of the budget set, there exists a positive $\epsilon$ such that the allocation $(1+\epsilon) \cdot y_{i}^{*}$ belongs to the budget set and has higher utility than $x_{i}^{*}$. In this case, $x^{*}$ would not be an equilibrium allocation, and we would have a contradiction. Therefore, $y_{i}^{*}$ is not in the interior of the budget set, that is: $z_{i}=x_{i}^{*}-y_{i}^{*}$ is such that $p^{*} \cdot z_{i}=0$.

QED 
With agents obtaining the same utility in states that they do not distinguish, prudent expected utility is equal to expected utility, for any prior probabilities over states of nature consistent with the given prior probabilities over observed events.

$$
\sum_{A_{i}^{j} \in P_{i}} \operatorname{Pr}_{i}\left(A_{i}^{j}\right) \min _{\omega \in A_{i}^{j}} u_{i}^{j}\left(x_{i}(\omega)\right)=\sum_{\omega_{s} \in \Omega} q_{i}^{s} u_{i}^{s}\left(x_{i}^{s}\right) .
$$

The pair $\left(y^{*}, p^{*}\right)$ is also a Walrasian equilibrium with prudent expectations. But, since $y^{*}$ gives the same utility in states that agents do not distinguish, the prudent behavior is not shown to have been unjustified. It never happens that an agent was expecting the worst and got something better. Prudent expectations are, in a certain sense, self-fulfilled. This constitutes a natural refinement of the concept of equilibrium.

A pair $\left(y^{*}, p^{*}\right)$ is a "prudent expectations equilibrium" if $p^{*}$ is a price system and $y^{*}=\left(y_{1}^{*}, \ldots, y_{n}^{*}\right)$ is a feasible allocation such that, for every $i, y_{i}^{*} \in \mathbb{R}_{+}^{\Omega l}$ maximizes $U_{i}$ on $B_{i}\left(p^{*}, e_{i}\right)$ with $u_{i}\left(y_{i}^{*}\right)$ being $P_{i}$-measurable.

Corollary 1 Given any Walrasian equilibrium with prudent expectations, $\left(x^{*}, p^{*}\right)$, there exists a prudent expectations equilibrium, $\left(y^{*}, p^{*}\right)$ under the same price system ( $y^{*}$ as defined in Theorem 1$)$.

Proof. The allocation $y^{*}$ has the same prudent expected utilities as the equilibrium allocation $x^{*}: U_{i}\left(y^{*}\right)=U_{i}\left(x^{*}\right)$, for all $i$. Under the price system $p^{*}$, both allocations cost the same: $p^{*} \cdot y_{i}^{*}=p^{*} \cdot x_{i}^{*}$, for all $i$. Thus, $y^{*}$ is also allowed by each agent's budget restriction, and maximizes utility. Furthermore, since $y^{*} \leq x^{*}, y^{*}$ is feasible.

QED

An important consequence is the existence of equilibrium allocations with $P_{i^{-}}$ measurable utility. If instead of restricting agents to consume the same in states that they do not distinguish, we restrict them to consume bundles with the same utility, equilibrium existence is guaranteed.

Corollary 2 There exists a prudent expectations equilibrium. 
There exists a Walrasian equilibrium of the Arrow-Debreu economy, so this is an obvious consequence of Corollary 1.

From Theorem 2, it is straightforward that if the contingent commodities have strictly positive prices, $p^{*}>>0$, then $z=0$ and $x^{*}=y^{*}$. That is, all Walrasian equilibria with prudent expectations have $P_{i}$-measurable utility. A condition that guarantees strict positivity of the prices of the contingent commodities is sufficient to guarantee that all Walrasian equilibria with prudent expectations are prudent expectations equilibria. For example, if every state is observed by at least one agent.

\section{Measurability implies incentive compatibility}

A measurability restriction is still present. In states of nature that agents do not distinguish, they are entitled to receive the same lists. The impossibility of observing the state of nature is not a problem. The agent does not fear being "tricked", because her rights are the same in states that she does not distinguish: to receive one of the bundles in the list.

In sum, the consideration of contracts for uncertain delivery allows us to relax in a natural way the measurability assumption, while preserving incentive compatibility. This enlarges the space of allocations, improving the efficiency of exchange, relatively to economies in which consumption has to be measurable with respect to private information.

\section{Welfare improves}

Compared with measurable consumption, measurable utility is less restrictive, as it allows agents to select different consumption bundles in states that they do not distinguish. As a result, they take advantage of the variations in prices across these states. The following theorem describes this and confirms that the cheapest bundle is chosen for delivery.

Theorem 3 Let $\left(x^{*}, p^{*}\right)$ be a Walrasian equilibrium with prudent expectations. 


$$
\omega^{\prime} \in P_{i}(\omega) \Rightarrow p^{*}(\omega) \cdot x_{i}^{*}(\omega) \leq p^{*}(\omega) \cdot x_{i}^{*}\left(\omega^{\prime}\right)
$$

Proof. Suppose that for some $\omega^{\prime} \in P_{i}(\omega)$, we had $p^{*}(\omega) \cdot x_{i}^{*}(\omega)>p^{*}(\omega) \cdot x_{i}^{*}\left(\omega^{\prime}\right)$. Designate by $y_{i}$ a modified bundle with $y_{i}^{*}(\omega)=x_{i}^{*}\left(\omega^{\prime}\right)$ being the only difference relatively to $x_{i}^{*}$. This bundle has the same utility and allows the agent to retain some income. There exists a positive $\epsilon$ such that $(1+\epsilon) \cdot y_{i}$ belongs to the budget set and has higher utility than $x_{i}^{*}$. Contradiction!

In spite of the penalization implied by prudence, prudent expected utility is higher in the sense of Pareto than that which is attainable under the classical restriction of equal consumption in states of nature that are not distinguished.

Theorem 4 Let $\left(x^{*}, p^{*}\right)$ be an equilibrium in the sense of Radner (1968).

There are Pareto optima of the economy with uncertain delivery, $z$, such that $U_{i}\left(z_{i}\right) \geq U_{i}\left(x_{i}^{*}\right)$, for every agent. The improvement may be strict (see section 3).

Proof. The proof is straightforward. If $\left(x^{*}, p^{*}\right)$ is an equilibrium in the sense of Radner (1968), the allocation $x^{*}$ is still feasible in the economy with prudent expectations.

From this result it does not follow that a prudent expectations equilibrium represents a Pareto-improvement relatively to the Walrasian expectations equilibrium. This is always true if equilibrium prices are the same. 


\section{Cooperative Solutions: the Prudent Cores}

In a cooperative setting it seems more difficult to justify that agents cannot reach the incentive compatible solution. Again, in a context of Knightian uncertainty a solution based on prudence and uncertain delivery can make sense. Conceptually, it may be placed between the private core (Yannelis, 1991) and the incentive compatible core.

Restricting our analysis to the case of prudent expectations, the core concept follows from the analysis in section 5 . The economy with uncertain delivery and prudent expectations was formalized as an Arrow-Debreu economy. The core of this Arrow-Debreu economy is the "prudent core".

A coalition $S \subseteq N$ "prudently blocks" an allocation $x$ if there exists $\left(y_{i}\right)_{i \in S}$ such that: $\quad \sum_{i \in S} y_{i} \leq \sum_{i \in S} e_{i}$ and $U_{i}\left(y_{i}\right)>U_{i}\left(x_{i}\right)$ for every $i \in S$, where $U_{i}$ is the prudent expected utility of agent $i$.

The "prudent core" is the set of all feasible allocations which are not prudently blocked by any coalition. Although coalitions of agents are formed, information is not shared between them. The prudent expected utility is based only on each agent's private information.

The prudent core is similar to a private core in which measurable utility is required instead of measurable consumption. An allocation without $P_{i}$-measurable utility may belong to the prudent core, but then there exists another allocation with the same utility for every agent, which has $P_{i}$-measurable utility and requires less resources.

Theorem 5 Let $x \in P C(\mathcal{E})$. There exists some $x^{\prime} \in P C(\mathcal{E})$ s.t., $\forall i=1, \ldots, n$ :

a) $x_{i}^{\prime} \leq x_{i}$;

b) $U_{i}\left(x_{i}^{\prime}\right)=U_{i}\left(x_{i}\right)$;

c) $u_{i}\left(x_{i}^{\prime}\right)$ is $P_{i}$-measurable. 
Proof. If $u_{i}\left(x_{i}\right)$ isn't $P_{i}$-measurable, we can multiply the $x_{i}(\omega)$ that have higher utilities in each element of $P_{i}$ by a factor smaller than 1 to obtain a modified allocation with measurable utility. These higher utilities are not taken into account in the calculation of prudent expected utility, because only the worst outcome is considered. Therefore, expected utility remains unchanged and this allocation satisfies $x_{i}^{\prime} \leq x_{i}$.

QED

Even being penalized by the prudence, allocations in the prudent core dominate, in the sense of Pareto, those in the private core (Yannelis, 1991). The latter are always feasible in the economy with uncertain delivery, while the converse is not true.

It is also possible to define a "prudent coarse core" and a "prudent fine core". In the prudent coarse core, prudence is based on common information.

A coalition $S \subseteq N$ strongly prudently blocks an allocation $x$ if there exists $\left(y_{i}\right)_{i \in S}$ such that: $\sum_{i \in S} y_{i} \leq \sum_{i \in S} e_{i}$ and $U_{i, S}^{s}\left(y_{i}\right)>U_{i S}^{s}\left(x_{i}\right)$ for every $i \in S$, where $U_{i S}^{s}$ is the "strongly S-prudent expected utility" of agent $i$ in the coalition $S$. The interim utility for agent $i$ in coalition $S$ is calculated using the minimum utility across states that the coalition cannot distinguish using only the common information among the members.

Analogously, the prudent fine core is based on pooled information.

A coalition $S \subseteq N$ weakly prudently blocks an allocation $x$ if there exists $\left(y_{i}\right)_{i \in S}$ such that: $\sum_{i \in S} y_{i} \leq \sum_{i \in S} e_{i}$ and $U_{i, S}^{w}\left(y_{i}\right)>U_{i S}^{w}\left(x_{i}\right)$ for every $i \in S$, where $U_{i S}^{w}$ is the "weakly S-prudent expected utility" of agent $i$ in the coalition $S$. The interim utility for agent $i$ in coalition $S$ is calculated using the minimum utility across states that the coalition cannot distinguish using the pooled information of its members.

In any case, welfare is improved in the prudent cores with respect to the definitions in which $P_{i}$-measurability is required. On the other hand, the welfare of the incentive compatible core cannot be reached in general. 


\section{Concluding Remarks}

In the presence of private information, general equilibrium theory in lines of the article of Radner (1968) cannot cope with widely used contracts, like options and contracts with default. In this paper, objects of choice are lists of bundles out of which the market selects one for delivery. This notion encompasses that of of objects of choice as contingent consumption claims (Arrow, 1953), allowing the study of more complex trade environments.

Under the assumption that agents have an extremely defensive behavior, the model of Arrow-Debreu can be reinterpreted to cover this situation. Therefore, many classical results still hold: existence of core and equilibrium, core convergence, continuity properties, etc. Expecting to receive the worst of the possibilities contracted, agents select bundles with the same utility for consumption in states that they do not distinguish. This improves welfare relatively to allocations with measurable consumption.

This equilibrium notion based on prudent behavior does not require agents to have any information about the characteristics of the other agents. This is the fundamental advantage relatively to the approach based on incentive compatibility. It is liable to criticism in contexts where agents have some information about the characteristics of others and could use it to construct their expectations.

There are situations in which prudence is reasonable, even when agents are informed about the characteristics of others. If there is a second round of trade, agents should evaluate portfolios by their indirect utility. In this case, the seller always delivers the bundle with the lowest value.

While an intuition for the concept of rational expectations equilibrium was the idea that "agents cannot be fooled", in the prudent expectations equilibrium it is the market that cannot be fooled. Agents use a rule of thumb which is related to Murphy's law: "if anything can go wrong, it will". 
Whether the agents know or not the state space of the economy is an important issue for the comparison between the two concepts. In this paper, agents do not have information about the state space and therefore cannot evaluate whether a contract in incentive compatible or not. As a result, their welfare falls short to that attained when all the possible incentive compatible trades are considered.

Agents with rational expectations know the space of states and are able to calculate correctly the probability of receiving each of the bundles in a list. But real economic agents follow simple rules of decision, instead of making huge amounts of calculations (Tversky and Kahneman, 1974). This further justifies the study of equilibrium with agents constructing expectations in a simple way.

\section{References}

Arrow, K.J. (1953), "The Role of Securities in the Optimal Allocation of Risk-Bearing", Econometrie, translated and reprinted in 1964, Review of Economic Studies, Vol. 31, pp. 91-96.

Arrow, K.J. and G. Debreu (1954), "Existence of an Equilibrium for a Competitive Economy", Econometrica, 22 (3), pp. 265-290.

Arrow, K.J. and L. Hurwicz (1972), "An Optimality Criterion for Decision Making Under Ignorance", In Uncertainty and Expectations in Economics: Essays in Honor of G.L.S. Shackle, ed. C.F. Carter and J.L. Ford. Oxford: Basil Blackwell.

Debreu, G. (1959), "Theory of Value", Wiley, New York.

Gilboa, I. and D. Schmeidler (1989), "Maxmin expected utility with non-unique priors", Journal of Mathematical Economics, 18, pp. 141153.

Harsanyi, J.C. (1967), “Games with Incomplete Information Played by 'Bayesian' Players", Parts I-III, Management Science, Vol. 14 (3, 5, 7), pp. 159-182, 320-324, 486-502.

Hildenbrand, W. and J.F. Mertens (1972), "Upper Hemi-Continuity of the EquilibriumSet Correspondence for Pure Exchange Economies", Econometrica, 40, pp. 99-108.

Holmström, B. and R.B. Myerson (1983), "Efficient and Durable Decision Rules with Incomplete Information", Econometrica, 51 (6), pp. 1799-1819. 
Klibanoff, P., M. Marinacci and S. Mukerji (2005), "A Smooth Model of Decision Making Under Ambiguity", Econometrica, 73 (6), pp. 1849-1892.

Postlewaite, A. and D. Schmeidler (1986), "Implementation in Differential Information Economies", Journal of Economic Theory, 39 (1), pp. 14-33.

Prescott, E.C. and R.M. Townsend (1984a), "Pareto Optima and Competitive Equilibria with Adverse Selection and Moral Hazard", Econometrica, 52 (1), pp. 21-46.

Prescott, E.C. and R.M. Townsend (1984b), "General Competitive Equilibria Analysis in an Economy with Private Information", International Economic Review, 25 (1), pp. $1-20$.

Radner, R. (1968), "Competitive Equilibrium under Uncertainty", Econometrica, 36 (1), pp. 31-58.

Schmeidler, D. (1989), "Subjective Probability and Expected Utility without Additivity", Econometrica, 57 (3), pp. 571-587.

Tversky, A. and D. Kahneman (1974), "Judgement under uncertainty: heuristics and biases", Science, 185, pp. 1124-1131.

Von Neumann, J. and O. Morgenstern (1944), "Theory of Games and Economic Behavior", Princeton University Press, Princeton.

Wilson, R. (1978), "Information, Efficiency, and the Core of an Economy", Econometrica, 46 , pp. 807-816.

Yannelis, N.C. (1991), "The Core of an Economy with Differential Information", Economic Theory, 1, pp. 183-198. 